\title{
Las publicaciones médicas y científicas en la era de la COVID-19
}

\author{
Medical and scientific publications in the COVID-19 era
}

\author{
Ricardo Figueroa-Damián* \\ Editor, Revista de Perinatología y Reproducción Humana, Ciudad de México, México
}

El año 2020 se ha caracterizado por el surgimiento y propagación de la pandemia de enfermedad por coronavirus 2019 (COVID-19), la cual representa uno de los mayores problemas de salud pública al que se ha enfrentado la humanidad. Hasta el momento, la infección ocasionada por el coronavirus 2 del síndrome respiratorio agudo grave (SARS-CoV-2), ha impactado no solo en el ámbito de la salud, sino prácticamente todas las actividades de la sociedad, incluyendo las áreas económica, laboral, familiar y educativa.

El sector de la salud, de manera natural, es una de las principales áreas involucradas en la atención de los problemas generados por la COVID-19: implementación de las medidas adecuadas de prevención, reconocimiento de los grupos poblacionales de mayor riesgo, identificación de los factores que incrementan el impacto de la infección, atención médica de los individuos enfermos y, de manera muy especial, desarrollo de proyectos de investigación encaminados a conocer con la mayor profundidad posible los fenómenos involucrados en origen y el desencadenamiento de la pandemia.

Es evidente que, para poder resolver un problema, el primer paso a dar es conocer dicho problema. La infección por el SARS-CoV-2 constituye una nueva entidad clínica, una nueva enfermedad, por lo que el conocimiento que tenemos de ella es muy limitado, prácticamente nulo y mientras persista la información restringida, no es posible que se propongan medidas efectivas para el control de la pandemia. De aquí la importancia de la investigación; es a partir de los resultados obtenidos y los descubrimientos logrados por los diferentes proyectos de investigación, que se irán desentrañando y entendiendo los fenómenos no conocidos, con lo que paulatinamente se pueden ir resolviendo las dudas del conocimiento que se investigan. En el caso específico de la COVID-19, la información que progresivamente van aportando los múltiples estudios científicos y médicos que se están llevando a cabo, en un futuro, esperamos pronto, hará que se cuente con el conocimiento suficiente de la enfermedad para controlarla.

Los resultados de las investigaciones, una vez que se van obteniendo, necesitan ser difundidos. La información que no se propaga es información que no existe. Es en esta parte donde adquieren relevancia las revistas de difusión científica y médica. Estos instrumentos bibliográficos representan la principal vía de difusión de los nuevos conocimientos generados por los investigadores. Por lo que respecta a la COVID-19 hasta el momento, la investigación científica ha permitido, en un tiempo extremadamente reducido, identificar al agente etiológico de esta nueva enfermedad: el SARS-CoV-2, con la descripción de sus características estructurales y de los componentes que le permiten interactuar con las células humanas. Así mismo ha sido posible establecer los

\section{Correspondencia:}

*Ricardo Figueroa-Damián

E-mail: rfd6102@yahoo.com.mx licencia CC BY-NC-ND (http://creativecommons.org/licenses/by-nc-nd/4.0/).

Fecha de recepción: 03-12-2021

Fecha de aceptación: 03-12-2021 DOI: 10.24875/PER.M21000008
Disponible en internet: 28-12-2021 Perinatol Reprod Hum. 2021;35(1):1-2 www.perinatologia.mx 
mecanismos fisiopatológicos relacionados con el desarrollo de la enfermedad. La aplicación de la información proporcionada por las diversas investigaciones que se están llevando a cabo ha permitido que en pocos meses se hayan desarrollado vacunas con alta potencialidad de eficacia en la prevención de la infección y en reducción de la severidad de la COVID-19.

Las revistas y publicaciones científicas y médicas vienen a jugar un papel de suma importancia en la difusión del conocimiento generado por los procesos de investigación, haciendo llegar a la comunidad médica y científica los nuevos conocimientos generados por las investigaciones de un número muy amplio de grupos de estudio e investigación. Es en este sentido que la revista Perinatología y Reproducción Humana pretende contribuir en la lucha contra la infección por el SARS-CoV-2, aportando sus páginas para la publicación de artículos de todos los campos que involucran la salud reproductiva. Hacemos la invitación a la comunidad médica y científica para que consideren a nuestra revista como uno de los órganos de difusión de sus investigaciones, y que de esta manera todos podamos aportar un pequeño grano de arena en la lucha contra la pandemia de COVID-19. 